\title{
A Novel Hybrid 2D/Q-2D Finite Element Method for Power/Ground Plane Analysis
}

\author{
Ping $\mathrm{Li}^{1}$, Li Jun Jiang ${ }^{1}$, and Hakan Bagci ${ }^{2}$ \\ ${ }^{1}$ Department of Electric and Electronic Engineering, The University of Hong Kong, Pokfulam Road, Hong Kong \\ Email: liping@eee.hku.hk, jiangli@,hku.hk \\ ${ }^{2}$ Division of Computer, Electrical, and Mathematical Sciences and Engineering, King Abdullah University of Science and Technology \\ (KAUST), Thuwal, 23955-6900, Saudi Arabia.
}

\begin{abstract}
By realizing the TMz modal distribution property between the power/ground plate-pairs, the magnetic field actually has only azimuthal components while longitude component is zero. Thus, if the magnetic field wave equation is solved, only a Quasi-2D issue is considered. Furthermore, since the high-order modes are only located in the near region of the anti-pad, thus for the domain far-away from the antipad has only the TEM mode. Thereby, in this region, the magnetic field is invariable along the vertical direction, which consequently is a pure $2 \mathrm{D}$ domain. As a result, the conventional $3 \mathrm{D}$ computational domain is naturally transformed to a hybrid 2D/Q-2D domain, which will significantly reduce the computational cost.
\end{abstract}

\section{INTRODUCTION}

The continuously rising clock frequency will cause significant electromagnetic interference (EMI) on the power supply vias crossing the power/ground planes, which could result in significant signal and power integrity related issues. To avoid the design failure at the early stage, it is very necessary to fully understand the related EM issues.

One strategy is based on the equivalent circuit model [1], which the interactions between via, plate, and antipad are equivalently represented by suitable coupling capacitors. This type of method is efficient, but could lose of accuracy. Another treatment is on the basis of full wave approach [2]-[3], which is very accurate since the full couplings among massive vias are included. However, the already proposed methods are still computational expensive.

In this work, a novel full-wave finite-element timedomain method is proposed. Based on the 2D and Quasi-2D (Q-2D) field distribution property, the original 3D domain will be simplified as a hybrid 2D and Q-2D domain. Thus, the computational cost will be significantly reduced.

\section{METHODOLOGY}

For a general power ground plate-pair, it is well known that the domain mode between the plate-pair is $\mathrm{TMz}$ mode, thus the vertical component along the thickness direction of the plate is null, and only the azimuthal components need to be considered. To fully make use of this property, the 3D triangular prism elements are employed as the basic mesh cells. For the Q-2D problem, the unknowns are merely located over the surfaces. In fact, the computational cost can be further reduced since the higher-order modes are highly confined in the proximity of antipads. The higher-order modes will be exponentially attenuated along the propagation direction, for region that is far-away from the anti-pad, only the fundamental mode is required. In this way, the 3D domain will be decomposed into a hybrid 2D and Q-2D domain, which will dramatically make the computation much more cheaper.

\section{RESULTS AND DISCUSSION}

To show the effectiveness of the proposed algorithm, a power/ground plate-pair having a rectangular antipad is studied. The thickness of the plate-pair is $0.254 \mathrm{~mm}$, and the relative dielectric permittivity is 2.2 . The separation distance between the $2 \mathrm{D}$ and $\mathrm{Q}-2 \mathrm{D}$ region is equal to the thickness of the plate-pair, namely, $0.254 \mathrm{~mm}$. In Fig. 1, the 2D (blue ones) and Q-2D (red ones) meshes are given. As can be seen, most the meshes belong to 2D domain, specifically, the number of $2 \mathrm{D}$ meshes is 4539 , and the number of meshes in the Q-2D domain is 1407 . The total number of unknowns for the hybrid method is 9718 , and the total number of unknowns for the 3D method is 19252. Thus, it is clearly noted that the hybrid method is much more efficient than the conventional one. For the accuracy of the proposed algorithm, we will present them in the conference.

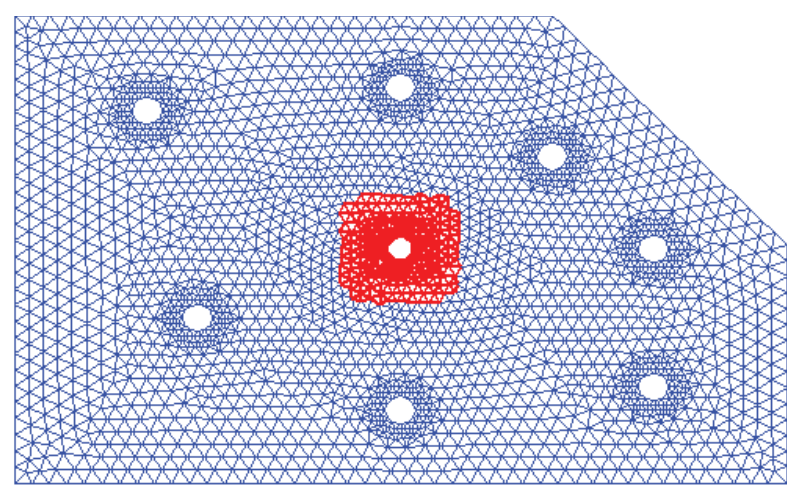

Fig. $12 \mathrm{D}$ and Q-2D meshes of the power/ground plate-pair.

\section{REFERENCES}

[1] Y. J. Zhang and J. Fan, "An intrinsic circuit model for multiple vias in an irregular plate pair through rigorous electromagnetic analysis," IEEE Trans. Microw. Theory Tech., vol. 58, no. 8, pp. 2251-2265, Aug. 2010.

[2] P. Li, L. J. Jiang, and H. Bagci, 'Tansient analysis of dispersive power-ground plate-pairs by DGTD method with wave port excitation," IEEE Trans. Electromagn. Compat., vol. 59, no. 1, pp. 172-183, Feb. 2017

[3] P. Li, L. J. Jiang, and H. Bagci, "Discontinuous Galerkin timedomain analysis of power-ground planes taking into account decoupling capacitors," IEEE Trans. Components, Packaging, and Manufacturing Techn., vol. 7, no. 9, pp. 1476-1485, Sept. 2017. 